\title{
Effect of Three Organic Solvents on Dewaxing of Wheat Straw
}

\author{
Guizhen Gong, Gaojun Sun
}

(School of Chemical Engineering, Xuzhou University of technology, Xuzhou 221018, Jiangsu

Province )

\begin{abstract}
The waxes in wheat straw were removed by ethanol, acetone and petroleum ether, and the extracts at all levels were analyzed by fourier transform infrared (FTIR). The results showed that the effect of dewaxing of ethanol was higher than that of acetone, and the effect of dewaxing of petroleum ether was the worst. The effects of the three solvents on the extraction of substances with different functional groups are different. The substance containing - $\mathrm{OH}$ can be extracted with three solvents, while the extraction of benzene ring and $-\mathrm{C}=\mathrm{O}$ with petroleum ether is better. The study has an important basic theoretical significance in the development of high added value of wheat straw.
\end{abstract}

Keywords: Wheat straw; Organic solvent; Dewaxing; Fourier transform infrared analysis

\section{三种有机溶剂对麦秆的脱蜡效果}

\author{
宫贵贞，孙高军
}

(徐州工程学院 化学化工学院, 江苏省 徐州市 221018)

摘要: 利用乙醇、丙酮、石油醚三种溶剂对小麦秸秆中的蜡进行脱除, 并用红外对各级萃取物进行分析。结果表明: 乙 醇脱蜡效果高于丙酮, 而石油醚脱蜡效果最差。三种溶剂对含有不同官能团的物质萃取效果有所不同, 三种溶剂均可对含- $0 \mathrm{H}$ 的物质进行萃取, 而对含有苯环和 $-\mathrm{C}=0$ 的物质, 石油醚的萃取效果较好。该研究在开发麦秆的高附加值利用方面具有重要 的基础理论意义。

关键词: 麦秆; 有机溶剂; 脱蜡; 红外分析

中图分类号: TQ353.9 文献标志码: A

引言

秸秆在我国产量巨大，但目前在我国作为资源利用的量较低，且利用方式还很落后，主要是直接燃烧 [1]。这种方式不仅效率低下, 浪费资源, 还造成了严重的环境污染。秸秆表面有一层蜡质层, 这层蜡质 的存在阻碍了水和有机溶剂在秥秆样品中的渗透, 不利于对秸秆后续的转化研究 [2-7]。蜡被广泛应用于 制造皮鞋油、地板蜡、复写纸等, 符合医药食品用蜡要求, 可以用于化妆、食品的生产及水果蔬菜的涂蜡 $[8,9]$ 。蜡由于用量较大, 不能满足人们的需要。若能从秸秆中提取蜡, 不仅可降低其成本, 还可提高秸 秆的利用价值 [10]。因此, 本文利用石油醚、乙醇、丙酮三种有机溶剂对麦秆的脱蜡进行了研究, 比较了 三种溶剂的脱蜡效果。

\section{1 实验部分}

\section{1 原料、试剂和仪器}

实验选用江苏徐州地区的小麦秸秆作为研究秥秆。将秸秆用去离子水清洗后自然风干, 用多功能粉碎 
机粉碎至过 200 目耖, 并在 $100{ }^{\circ} \mathrm{C}$ 下真空干燥 $5 h$, 取出置于干燥器内保存备用。

所用试剂石油醚（上海苏化学试剂有限公司）、乙醇（天津市福晨化学试剂有限公司）、丙酮（上海试 四赫维化工有限公司）均为分析纯试剂, 所有有机溶剂均经旋转蒸发仪蒸馏后使用。上海亚荣生化仪器厂 生产的 RE52CS-1 型旋转蒸发仪; ALPHA 型傅立叶转换红外光谱仪 (FTIR，德国 Bruker 公司)。

\section{2 实验方法}

用电子天平称取干燥的麦秆 $1.5 \mathrm{~g}$, 放入索式提取器中, 并加入 $170 \mathrm{~mL}$ 的 $95 \%$ 乙醇, 加热套加热, 连续萃取 $5 h$ 。待冷凝液刚刚虹吸下去时, 立即停止加热。冷至室温, 用旋转蒸发仪蒸馏, 回收提取液中 的大部分乙醇, 得到浓缩液, 立即装入标有乙醇 1 的瓶子中。第二次萃取前将上次的萃取残渣进行烘干, 并用电子天平称量其质量, 记录下来。然后再进行下一次的萃取，依次萃取 5 次。

石油醚和丙酮依相同的实验流程对小麦秸秆进行萃取, 每个溶剂分别连续萃取五次, 每次时间为 $5 h$ 。 所得各级萃取液用 FTIR 分析, 采用 KBr 压片, 在 $400^{\sim} 4000 \mathrm{~cm}-1$ 波数范围内进行扫描。

\section{2 结果与讨论}

\section{1 萃取过程分析}

石油醚、乙醇、丙酮三种萃取液的颜色，乙醇萃取液颜色最深，丙酮次之，颜色最浅的是石油梄萃取 液。

图 1 是三种溶剂 5 次分级萃取液的质量。由图可见, 三种溶剂的萃取量均呈现先低到高, 再由高到低, 第三次萃取量均为最高。说明麦秆中可萃取物是逐渐溶出的过程。萃取过程是萃取剂对秥秆进行浸润, 渗 透, 膨胀的过程。在第三次萃取循环中, 萃取剂完全渗入, 与秸秆中小分子接触完全, 萃取剂的溶解能力 变强, 因而萃取效果达到最高。而后秥秆中的小分子越来越少，萃取量逐步减少，最终萃取完成。

三种溶剂总的萃取量，石油醚萃取质量小于丙酮萃取物质量，小于乙醇萃取质量。其原因为乙醇还有 极性官能团，根据相似相容原理，秸秆中的极性化合物更易溶于乙醇。

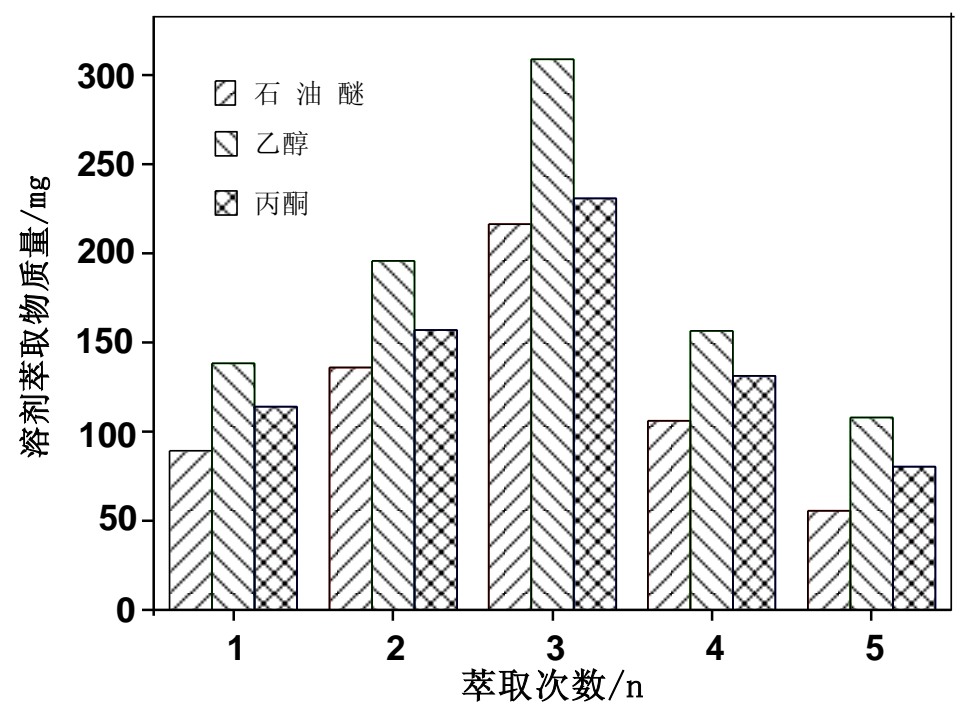

图 1 不同溶剂萃取物的质量

\section{2 红外分析}

图 2 4 分别为丙酮萃取物、石油醚萃取物及乙醇萃取物的 FTIR 谱图。图中所示，在 $3415 \mathrm{~cm}-1$ 是氢 
键缔合的 $-\mathrm{OH}$ 特征峰, 三种萃取液中, 该处的吸收峰均较强, 从第 1 次到第 5 次, 随着萃取的进行, 强度 均呈现减弱趋势。 $3000^{\sim} 2800 \mathrm{~cm}-1$ 为碳水化合物、木质素和脂肪族化合物中 CH3-和-CH2-的伸缩振动峰, 石油醚萃取物该区域吸收峰最强, 乙醇次之, 丙酮最弱, 且强度随着萃取次数的增多依次减弱, 第 5 次的 乙醇萃取物中已不能观察到此处吸收峰。 $1750 \mathrm{~cm}-1$ 为含 $\mathrm{C}=0$ 的吸收峰, 丙酮萃取物中在第 $1 、 3 、 5$ 次中 可观察到此峰, 且第 5 次最强; 石油醚萃取物中该吸收峰较强, 且随着萃取次数的增加, 呈现增加的趋势, 乙醇萃取物中该峰吸收不明显。说明含 $\mathrm{C}=0$ 基团的物质, 较易被石油醚提取, 且随时间的延长逐渐析出。 $1500 \mathrm{~cm}-1,1600 \mathrm{~cm}-1$ 是芳环的骨架 $\mathrm{VC}=\mathrm{C}$ 的振动特征吸收峰, 三种萃取物中，石油醚大于乙醇，丙酮最 弱。且石油醚及乙醇萃取液中的强度随萃取次数增加而减弱, 丙酮萃取液则呈现逐渐增加的趋势。

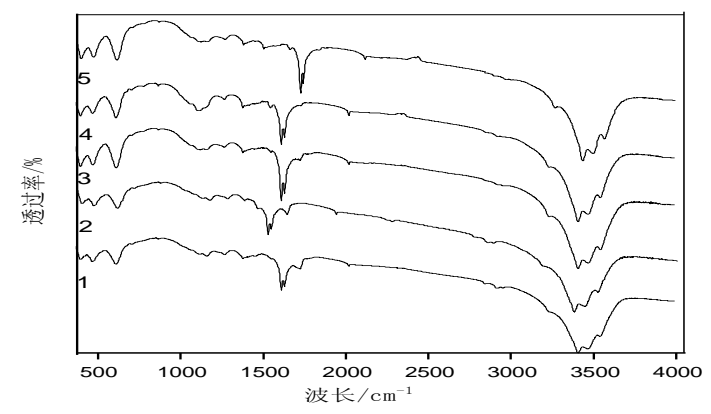

图 2 丙酮萃取液 FTIR

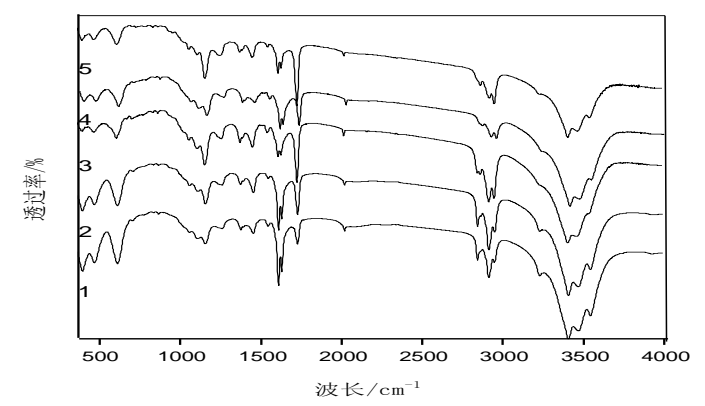

图 3 石油醚萃取液 FTIR

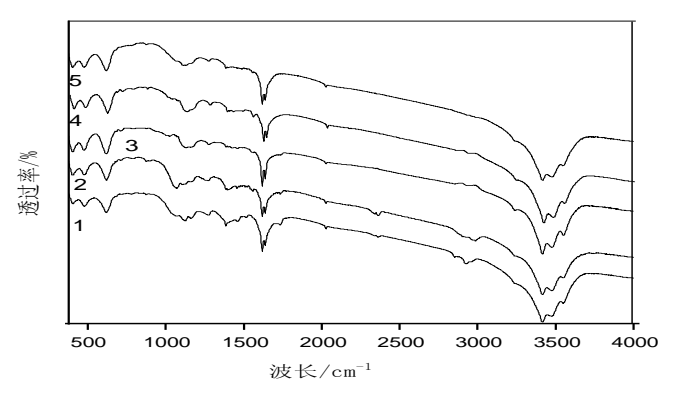

图 4 乙醇萃取液 FTIR

\section{3 结论}

本研究考察了丙酮、石油醚和乙醇对麦秆脱蜡的效果。乙醇的萃取率高于丙酮和石油醚，其原因可能 是乙醇为极性分子可以破坏麦秆的网络大分子，从而使一些蜡质成分被提取。FTIR 分析显示，三种溶剂对 含不同官能团的物质优先萃取特性有所不同, 三种溶剂均可对含 $-\mathrm{OH}$ 的物质进行萃取, 而对含有苯环和 $-\mathrm{C}=0$ 的物质，石油醚的萃取效果较好。该研究在开发麦秆的高附加值利用方面具有重要的基础理论意义。 


\section{4 致谢}

感谢中国建筑材料联合会（2014-M3-4)，徐州情报所课题（XKQ016）基金的资助

\section{Acknowledgement}

This work was supported by the China Building Material Federation (2014-M3-4) and Xuzhou Information Institute (XKQ016).

\section{参考文献:}

[1] 李兴平．浅析农作物秥秆的综合利用 [J]．洛阳理工学院学报(自然科学版)，2010，（3）：8-11.

[2］郭国进．酮苯脱蜡装置的工艺技术进展［J]．炼油与化工，2011，（6）：19-21.

［3］何濒，苏玉忠，谭思，等. 酮苯脱蜡中试装置和试验 [J]．厦门大学学报 (自然科学版)，2005，（3）：390-394.

[4] 李丙庚，王海燕．实验室快速模拟脱蜡方法的建立与应用 [J]。润滑油，2013，（5）：46-49.

[5］李松华，刘晓非，庄旭品，等. 棉浆泊纤维素的超声波处理 [J]．应用化学，2003，(11）：1030-1034.

［6］王彦芳．气相色谱法测定蜡烛中植物蜡的含量 [J]．当代化工，2013，(6)：879-880.

[7] 程赛，郡兴峰，倪穗，等．秋橄榄果实中番茄红素有机溶剂的萃取及其稳定性研究 [J]。食品与发酵工业，2011， (1) : 179-193.

[8］凡夫，育联．生物蜡的神奇美肤功效 [J]。医学美学美容，2001，(5)：88.

[9] 王有琼，段琼芬，赵虹，等．绿色产品一生物蜡 [J]。中国林副特产，2002，（3）：28-29.

[10］鞠守勇．作物秥秆资源开发与利用 [J]．广东化工，2014，(5)：126-127.

\section{References:}

[1] Li Xingping. Study on Comprehensive Utilization of Crop Straw [J]. Journal OF Luoyang Institute of technology (Natural Science), 2010, (3): 8-11.

[2] Guo Guojin. The progress of dewaxing device technology by ketone benzene [J]. Refining \& Chemical, 2011, (6): 19-21.

[3] He Hao, Su Yuzhong, Tan Si, et al. Pilot equipment and test of ketone benzene dewaxing [J]. Journal of Xiamen University (Natural Science Edition), 2005, (3): 390-394.

[4] Li Binggeng, Wang Haiyan. Establishment and application of rapid simulation of dewaxing method in laboratory [J]. Lubricating Oil, 2013, (5): 46-49.

[5] Li Song Wow, Liu Xiaofei, Zhuang Xuping, et al. Ultrasonic treatment of cellulose pulp [J]. Chinese Journal of Applied Chemistry, 2003, (11): 1030-1034.

[6] Wang Yanfang. Determination of plant wax content in candles by gas chromatography [J]. Contemporary Chemical Industry, 2013, (6): 879-880.

[7] Cheng Sai, Shao Xing-feng, Ni Sui, et al. Study on extraction and stability of lycopene organic solvent in autumn olive fruit [J]. Food and Fermentation Industry, 2011, (1): 179-193.

[8] Fan Fu, Yu Lian. The magic beauty effect of biological wax [J]. Medical Aesthetics Beauty, 2001, (5): 88.

[9] Wang Youqiong, Duan Qiongfen, Zhao Hong, etc. Green products - biological wax [J]. China Forest Deputy Products, 2002, (3): 28-29.

[10] Ju Shouyong. Development and Utilization of Crop Straw Resources [J]. Guangdong Chemical Industry, 2014, (5): 126-127. 